ESAIM: PROCEEDINGS, September 2005, Vol.14, 201-212

Eric Cancès \& Jean-Frédéric Gerbeau, Editors

DOI: $10.1051 /$ proc:2005015

\title{
OUTLET DISSIPATIVE CONDITIONS FOR AIR FLOW IN THE BRONCHIAL TREE*
}

\author{
B. Maury ${ }^{1}$, N. Meunier ${ }^{2}$, A. Soualah ${ }^{1}$ And L. Vial ${ }^{2}$
}

\begin{abstract}
We propose inflow and outflow boundary conditions to handle the airflow through the upper part of bronchial tree-like domains. This approach is based on a condensation of the part of the domain which is geometrically complex by using the linearity of the flow through a net of ducts with small diameters with respect to the pressure jumps. We show that the boundary conditions we obtain lead to a well-posed problem, and we present some numerical tests where the results with those new conditions are compared with the results obtained by simulating the flow over the full (non-condensed) domain.
\end{abstract}

\section{IntRoduction, Motivation}

This work is motivated by the research of numerical methods to simulate airflow in the whole human tracheobronchial tree from the trachea to the 23rd generation. The main idea is to perform direct simulations in the proximal airways and to take into account the downstream pulmonary ramifications by using an appropriate dissipative boundary condition. In previous works related to flow in airways, outlet and inlet boundary conditions were commonly Dirichlet boundary conditions, see for exemple [6]. Other conditions for the Navier-Stokes equations are described in [1] and [7], where pressure and flow rate are imposed. In haemodynamics, we mention in particular [3] and [8], where simulations of flow in blood vessels are performed, based on boundary conditions involving flow rate and averaged pressure imposed on several domain sections. In our context, outlet boundary conditions are expected to meet the two requirements:

(1) any nonzero flow rate through the condensed part of the tree has to be paid by a loss of energy,

(2) the forcing term (the "engine") must be contained in those conditions (during the normal inspiration process, the air is pulled from downstream).

The two sets of boundary conditions we propose, based on modeling considerations, are designed to fill those expectations.

So, our aim is to model the flow of a viscous incompressible fluid that obeys Stokes or Navier-Stokes equations through a ramified domain of $\mathbb{R}^{2}$ or $\mathbb{R}^{3}$. The geometry under consideration is schematically represented in figure 1. Note that each subdomain $\tilde{\Omega}_{i}$ has itself a ramified dichotomous geometrical structure. Indeed, in the case of the bronchial tree, we can represent, without loss of generality each subdomain as a single pipe.

\footnotetext{
* This work has been supported by the ACI project LePoumonVousDisJe and by Air Liquide group.

${ }^{1}$ Laboratoire de Mathématiques, Université Paris-Sud, Bâtiment 425, 91405 Orsay Cedex, France;

e-mail: Bertrand.Maury@math.u-psud.fr \& Assia.Soualah@math.u-psud.fr.

2 Laboratoire Jacques-Louis Lions, Université Pierre et Marie Curie, Boîte courrier 187, 75252 Paris Cedex 05, France ;

e-mail: meunier@ann.jussieu.fr \& vial@ann.jussieu.fr.
}

(c) EDP Sciences, SMAI 2005 


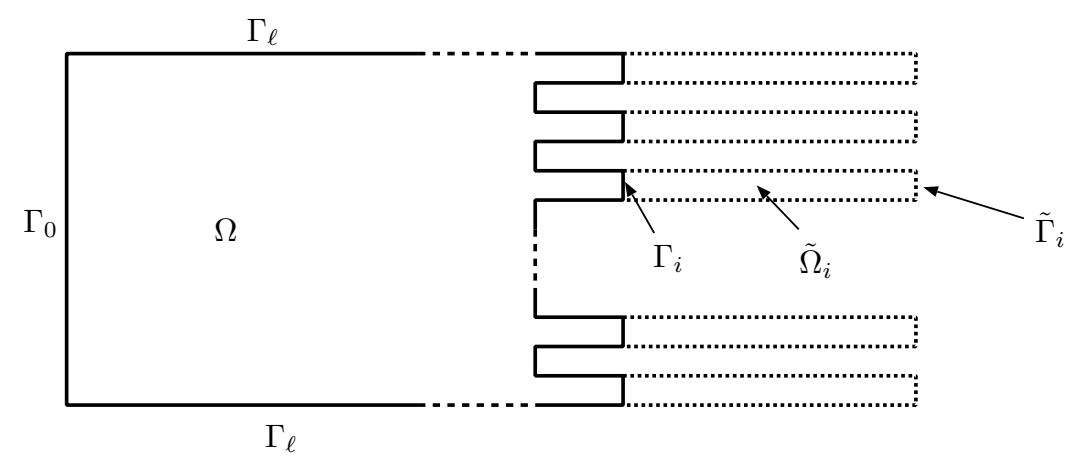

We assume that the airflow is related linearly to the difference of pressure between the "inlet" $\Gamma_{0}$ and the "outlets " $\tilde{\Gamma}_{i}$, for $i=1, \ldots, N$. Note that $\Gamma_{0}$ is a proper inlet during the inspiration phase only, but for a sake of simplicity, we will always use this terminology. Despite the geometrical complexity of the domain (the number of outlets is of order $2^{23}$ in the human lung), the flow in the small bronchia, thanks to the small diameter of the section of the pipe, is in fact governed by a very simple law. This law is analogous to the electrical relation in a network in which the flow rate is the intensity while the pressure is the electric potential. This analogy is described in details in the following section. The essential interest of this approach is to separate the fluid domain into a Navier-Stokes part (in which inertia of the fluid cannot be neglected) and a Stokes part, we will present here an approach based only on Stokes equations on the entire tree. A generalization to Navier-Stokes equations in $\Omega$ is then straightforward, from both the formulation and the numerical point of view.

\section{Models}

Our approach consists in "condensing" the downstream part of the domain by considering that the flow in each truncated part (the $\tilde{\Omega}_{i}$ ) is governed by a model of generalized Poiseuille's law (we do not make any assumption on the section of the pipes). We assume on the first hand that the pressure is uniform on the inlet $\Gamma_{i}$ (we denote $P_{i}$ its value), and on the other hand, that the flow in $\tilde{\Omega}_{i}$ is governed by Stokes equations with natural boundary conditions

$$
\mu \nabla \mathbf{u} \cdot \mathbf{n}-\mathbf{p} \mathbf{n}=-\Pi_{i} \mathbf{n}
$$

where the pressures $\Pi_{i}$ correspond to the pressure at the alveoli level associated to the branchus $i$ of the tree. Thanks to the linearity of the problem with respect to the jump of the pressure $P_{i}-\Pi_{i}$, there exists a constant $R_{i}$ ("resistance" of branch $i$ ) that does not depend on the geometry, such that

$$
P_{i}-\Pi_{i}=R_{i} \int_{\Gamma_{i}} \mathbf{u} \cdot \mathbf{n} .
$$

We now consider that the pressure $P_{i}$ is seen from the $\Omega$ side of the whole domain as a downstream pressure of $\Gamma_{i}$. We explicit this approach in the two following models.

\subsection{Natural dissipative Conditions}

In this first case, we simply write a free outlet condition on every outlet $\Gamma_{i}$ of the boundary. We eliminate the outlet pressure $P_{i}$ using relation (2). If we prescribe a natural condition (non dissipative) associated to an 
exterior pressure $P_{0}$, we obtain

$$
\left\{\begin{aligned}
-\mu \triangle \mathbf{u}+\nabla \mathrm{p} & =0 & & \text { in } \Omega \\
\nabla \cdot \mathbf{u} & =0 & & \text { in } \Omega \\
\mu \nabla \mathbf{u} \cdot \mathbf{n}-\mathrm{p} \mathbf{n} & =-P_{0} \mathbf{n} & & \text { over } \Gamma_{0} \\
\mu \nabla \mathbf{u} \cdot \mathbf{n}-\mathrm{p} \mathbf{n} & =-\Pi_{i} \mathbf{n}-R_{i}\left(\int_{\Gamma_{i}} \mathbf{u} \cdot \mathbf{n}\right) \mathbf{n} & & \text { over } \Gamma_{i} \quad i=1, \ldots, N
\end{aligned}\right.
$$

\subsubsection{Variational formulation}

We consider the functional spaces, $V=\left\{u \in H^{1}(\Omega)^{d}, u=0\right.$ on $\left.\Gamma_{l}\right\}, d=2$ or 3 , for the velocity field, and $M=L^{2}(\Omega)$ for the pressure. Multiplying by a test fonction $\tilde{\mathbf{u}} \in V$, and integrating by parts, the variational formulation of the problem with natural dissipative conditions writes

$$
\left\{\begin{array}{l}
\text { find } \mathbf{u} \in V, \text { and } \mathrm{p} \in M \text { satisfying } \\
\mu \int_{\Omega} \nabla \mathbf{u}: \nabla \tilde{\mathbf{u}}+\sum_{i=1}^{N} R_{i}\left(\int_{\Gamma_{i}} \mathbf{u} \cdot \mathbf{n}\right)\left(\int_{\Gamma_{i}} \tilde{\mathbf{u}} \cdot \mathbf{n}\right)-\int_{\Omega} \mathrm{p} \nabla \cdot \tilde{\mathbf{u}} \\
=-P_{0} \int_{\Gamma_{0}} \tilde{\mathbf{u}} \cdot \mathbf{n}-\sum_{i=1}^{N} \Pi_{i} \int_{\Gamma_{i}} \tilde{\mathbf{u}} \cdot \mathbf{n} \quad \forall \tilde{\mathbf{u}} \in V \\
\int_{\Omega} \mathrm{q} \nabla \cdot \mathbf{u}=0 \quad \forall \mathrm{q} \in M
\end{array}\right.
$$

This problem is then of the type

$$
\left\{\begin{array}{l}
\text { find } \mathbf{u} \in V, \text { and } \mathrm{p} \in M \text { satisfying } \\
a(\mathbf{u}, \tilde{\mathbf{u}})+b(\tilde{\mathbf{u}}, \mathrm{p})=\langle f, \tilde{\mathbf{u}}\rangle \quad \forall \tilde{\mathbf{u}} \in V \\
b(\mathbf{u}, \mathrm{q})=0 \quad \forall \mathrm{q} \in M
\end{array}\right.
$$

where the bilinear forms $a$ and $b$ are respectively defined by

$$
a(\mathbf{u}, \tilde{\mathbf{u}})=\mu \int_{\Omega} \nabla \mathbf{u}: \nabla \tilde{\mathbf{u}}+\sum_{i=1}^{N} R_{i}\left(\int_{\Gamma_{i}} \mathbf{u} \cdot \mathbf{n}\right)\left(\int_{\Gamma_{i}} \tilde{\mathbf{u}} \cdot \mathbf{n}\right), b(\mathbf{u}, \mathrm{p})=-\int_{\Omega} \mathrm{p} \nabla \cdot \tilde{\mathbf{u}}
$$

and the linear form $\langle f, \tilde{\mathbf{u}}\rangle=-P_{0} \int_{\Gamma_{0}} \tilde{\mathbf{u}} \cdot \mathbf{n}-\sum_{i=1}^{N} \Pi_{i} \int_{\Gamma_{i}} \tilde{\mathbf{u}} \cdot \mathbf{n}$.

\subsubsection{Existence and uniqueness}

We verify that the problem below has a unique velocity-pressure solution using the usual technique for the Stokes problem [5]. We design by $B \in L(M, V)$ the operator defined by $B \mathbf{u}=\nabla \cdot \mathbf{u}$, and we denote by $B^{\star}$ its adjoint. It suffices to verify that

(1) the bilinear forms $a$ and $b$ are continuous,

(2) the linear form $f$ is continuous,

(3) $a(\cdot, \cdot)$ is coercive,

(4) there exists $\beta>0$ such that, for all $\mathrm{q} \in M,\left\|B^{\star} \mathrm{q}\right\|>\beta\|\mathrm{q}\|$.

Points (1), (2), and (3) are straightforward. It remains to check the inf-sup condition. To show that $B^{\star}$ satisfies (4), it suffices to verify that the divergence operator is surjective from $V$ to $M$. We have then to prove 
that for all $\mathrm{q} \in M$, there exists $\mathbf{v} \in V$ such that $\mathrm{q}=\nabla \cdot \mathbf{v}$.

Consider $\mathrm{q} \in M$, there exists $\mathbf{v}_{1} \in H^{1}(\Omega)^{d}$ such that $\mathrm{q}=\nabla \cdot \mathbf{v}_{1}$. Consider $g=\mathbf{v}_{1 \mid \Gamma_{l}}$. We extend $g$ to $\tilde{g}$ such that $\tilde{g} \in H^{\frac{1}{2}}(\partial \Omega)$ and $\int_{\partial \Omega} \tilde{g} \cdot \mathbf{n}=0$. Under this condition, there exists (see $\left.[5]\right) \mathbf{w} \in H^{1}(\Omega)$ such that $\nabla \cdot \mathbf{w}=0$ and $\mathbf{w}_{\mid \partial \Omega}=\tilde{g}$. Let $\mathbf{v}=\mathbf{v}_{1}-\mathbf{w} \in H^{1}(\Omega)$. Then $\mathbf{v}$ verifies $\nabla \cdot \mathbf{v}=\nabla \cdot \mathbf{v}_{1}-\nabla \cdot \mathbf{w}=\mathrm{q}$ and $\mathbf{v}_{\mid \Gamma_{l}}=0$.

\subsection{Essential dissipative conditions}

We consider in this section more restrictive conditions, where the velocity profiles are imposed. $N+1$ velocity profiles $\mathbf{U}_{i}$, for $i=0, \ldots, N$, are given on $\Gamma_{0}, \ldots, \Gamma_{N}$, respectively, and we impose that there exists for all $i$ a real $\lambda_{i}$ (negative or positive depending on whether the fluid is coming in or out through $\Gamma_{i}$ ) such that $\mathbf{u}=\lambda_{i} \mathbf{U}_{i}$. These boundary conditions are

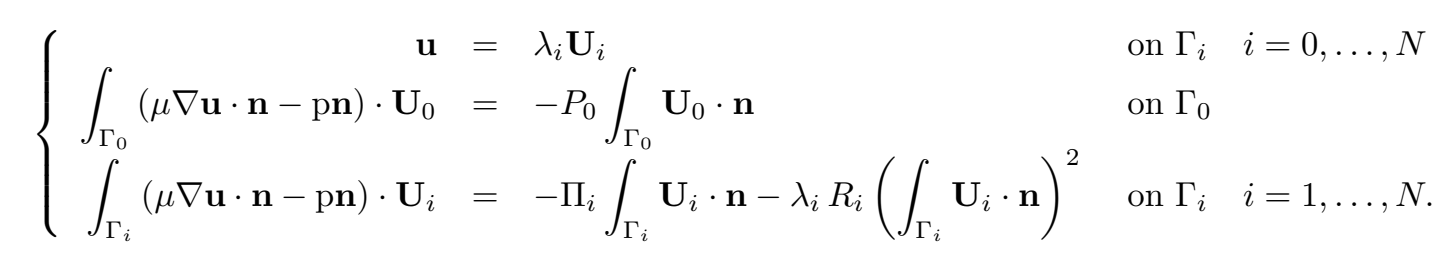

\subsubsection{Variational formulation}

We suppose that the velocity profiles $\mathbf{U}_{i}$ are given in $H^{1 / 2}\left(\Gamma_{i}\right)$. Pressures $P_{0}, \Pi_{1}, \ldots, \Pi_{N}$ are also given. We denote by $\Lambda=\left(\lambda_{0}, \ldots, \lambda_{N}\right)$ a vector of $R^{N+1}$, and by $H_{0}^{1}(\Omega)^{d}$ the space of functions of $H^{1}(\Omega)^{d}$ with a null trace on $\Gamma_{l}$. We introduce the functional spaces

$$
W=\left\{(\mathbf{u}, \Lambda) \in H_{0}^{1}(\Omega)^{d} \times R^{N+1}, \mathbf{u}=\lambda_{i} \mathbf{U}_{i} \text { over } \Gamma_{i} \quad i=0, \ldots, N\right\}, \text { and } M=L^{2}(\Omega) .
$$

The problem is then

$$
\left\{\begin{array}{l}
\text { find }(\mathbf{u}, \Lambda) \in W, \text { and } \mathrm{p} \in M \text { such that } \\
\mu \int_{\Omega} \nabla \mathbf{u}: \nabla \tilde{\mathbf{u}}-\int_{\Omega} \mathrm{p} \nabla \cdot \tilde{\mathbf{u}}+\sum_{i=1}^{N} R_{i}\left(\int_{\Gamma_{i}} \mathbf{U}_{i} \cdot \mathbf{n}\right)^{2} \lambda_{i} \tilde{\lambda}_{i} \\
=-P_{0} \tilde{\lambda}_{0} \int_{\Gamma_{0}} \mathbf{U}_{0} \cdot \mathbf{n}-\sum_{i=1}^{N} \tilde{\lambda}_{i} \int_{\Gamma_{i}} \mathbf{U}_{i} \cdot \mathbf{n} \quad \forall(\tilde{\mathbf{u}}, \tilde{\Lambda}) \in W \\
\int_{\Omega} \mathrm{q} \nabla \cdot \mathbf{u}=0 \quad \forall \mathrm{q} \in M .
\end{array}\right.
$$

The weak mixed formulation of the problem to solve is also of the type

$$
\left\{\begin{array}{l}
\text { find }(\mathbf{u}, \Lambda) \in W \text { and } \mathrm{p} \in M \text { such that } \\
a(\mathbf{u}, \tilde{\mathbf{u}})+b(\tilde{\mathbf{u}}, \mathrm{p})=\langle f, \tilde{\mathbf{u}}\rangle, \quad \forall(\tilde{\mathbf{u}}, \tilde{\Lambda}) \in W \\
b(\mathbf{u}, \mathrm{q})=0 \quad \forall \mathrm{q} \in M
\end{array}\right.
$$

with

$$
a(\mathbf{u}, \tilde{\mathbf{u}})=\mu \int_{\Omega} \nabla \mathbf{u}: \nabla \tilde{\mathbf{u}}+\sum_{i=1}^{N} R_{i}\left(\int_{\Gamma_{i}} \mathbf{U}_{i} \cdot \mathbf{n}\right)^{2} \lambda_{i} \tilde{\lambda}_{i}, b(\tilde{\mathbf{u}}, \mathrm{p})=-\int_{\Omega} \mathrm{p} \nabla \cdot \tilde{\mathbf{u}},
$$

and $\langle f, \tilde{\mathbf{u}}\rangle=-P_{0} \tilde{\lambda}_{0} \int_{\Gamma_{0}} \mathbf{U}_{0} \cdot \mathbf{n}-\sum_{i=1}^{N} \tilde{\lambda}_{i} \int_{\Gamma_{i}} \mathbf{U}_{i} \cdot \mathbf{n}$. 


\subsubsection{Existence and uniqueness}

We remark that the two models, both with natural and essential dissipative conditions, have the same variational formulation and differ only by the functional space correspending to the velocity field, and that $W \subset V$. Thus, to prove that the problem is well posed, it suffices to verify the surjectivity of the divergence operator from $W$ onto $M$.

Let $\mathrm{q} \in M$, and let $\Lambda=\left(\lambda_{0}, \ldots, \lambda_{N}\right) \in R^{N+1}$ be such that $\sum_{i=0}^{N} \int_{\Gamma_{i}} \lambda_{i} \mathbf{U}_{i} \cdot \mathbf{n}=\int_{\Omega} \mathrm{q}$. Consider $g$ such that $g=\lambda_{i} \mathbf{U}_{i}$ on $\Gamma_{i}, i=0, \ldots, N$, and $g=0$ on $\Gamma_{l}$. Since $g \in H^{\frac{1}{2}}(\partial \Omega)$, there exists $\mathbf{w} \in H^{1}(\Omega)^{d}$ such that $\mathbf{w}_{\mid \partial \Omega}=g$. Let $\mathrm{q}_{0}=\nabla \cdot \mathbf{w}-\mathrm{q}$. We have $\mathrm{q}_{0} \in L^{2}(\Omega)$ and

$$
\int_{\Omega} \mathrm{q}_{0}=\int_{\Omega} \nabla \cdot \mathbf{w}-\mathrm{q}=\int_{\partial \Omega} g \cdot \mathbf{n}-\int_{\Omega} \mathrm{q}=0,
$$

thus $\mathrm{q}_{0} \in L_{0}^{2}(\Omega)$ (set of $L^{2}$ functions with zero mean value). By virtue of the surjectivity of the divergence operator from $H_{0}^{1}(\Omega)^{d}$ in $L_{0}^{2}(\Omega)($ see $[5])$, there exists $\mathbf{w}_{0} \in H_{0}^{1}(\Omega)^{d}$ such that $\nabla \cdot \mathbf{w}_{0}=\mathrm{q}_{0}$. Then $\mathbf{v}=\mathbf{w}-\mathbf{w}_{0}$ verify $\nabla \cdot \mathbf{v}=\mathrm{q}, \mathbf{v}_{\Gamma_{l}}=0$, and $\mathbf{v}_{\Gamma_{i}}=\lambda_{i} \mathbf{U}_{i}, i=0, \ldots, N$.

\section{Global Resistances And pressures}

\subsection{Direct calculations of the equivalent resistances and pressures}

In this section, we concentrate on the dissipative region of the airway tree that we intend to condense in an appropriate boundary condition. In the case of a realistic airway tree, the dissipative part of the tracheobronchial tree would be located from the 6th to the 19th generation downstream the proximal airways which would be situated from the trachea down to the 5th generation. The flow is supposed to follow the Poiseuille's law in any of the bronchioles of the dissipative region of the airway tree. To study the flow behaviour in this condensed structure, we consider an airway tree of $K$ generations and denote by $P_{0}$ the inlet pressure, and by $P_{K}^{i}, 1 \leq i \leq 2^{K-1}$, the outlet pressures. For a bronchiole $i$ located at a generation $k, 1 \leq k \leq K$, we denote by $R_{k}^{i}$ its resistance and by $Q_{k}^{i}$ the flow rate through it. For $P_{0}$ and $P_{K}^{i}, 1 \leq i \leq 2^{K-1}$, given, the flow is completely described in the airway tree by the flow rate conservation law and Poiseuille's law in any bronchiole. In fact, solving this system leads to the following matricial formulation $R_{K} Q_{K}=P_{K}$, with $Q_{K}=\left[Q_{K}^{1}, \ldots, Q_{K}^{2^{K-1}}\right]^{T}$, $P_{K}=\left[P_{0}-P_{K}^{1}, \ldots, P_{0}-P_{K}^{2^{K-1}}\right]^{T}$ and $R_{K} \in M_{2^{K-1}}(\mathbb{R})$ having the particular following form that results from the superposition of block structure matrices like illustated below

$$
\begin{gathered}
R_{1}^{1} I_{K}^{1}+\left[\begin{array}{cc}
R_{2}^{1} I_{K}^{2} & 0 \\
0 & R_{2}^{2} I_{K}^{2}
\end{array}\right]+\left[\begin{array}{cccc}
R_{3}^{1} I_{K}^{3} & 0 & 0 & 0 \\
0 & R_{3}^{2} I_{K}^{3} & 0 & 0 \\
0 & 0 & R_{3}^{3} I_{K}^{3} & 0 \\
0 & 0 & 0 & R_{3}^{4} I_{K}^{3}
\end{array}\right] \\
+\cdots+\left[\begin{array}{ccc}
R_{k}^{1} I_{K}^{k} & \cdots & 0 \\
\vdots & \ddots & \vdots \\
0 & \cdots & R_{k}^{2^{k-1}} I_{K}^{k}
\end{array}\right]+\cdots+\left[\begin{array}{ccc}
R_{K}^{1} & \cdots & 0 \\
\vdots & \ddots & \vdots \\
0 & \cdots & R_{K}^{2^{K-1}}
\end{array}\right]
\end{gathered}
$$

with $I_{K}^{k} \in M_{2^{K-k}}(\mathbb{R})$ the one-rank matrix whose all entries are equal to 1 , for $1 \leq k \leq K$. We prove that this matrix that includes all the geometric parameters of the problem is symmetric, positive definite if $R_{k}^{i}>0$, $1 \leq i \leq 2^{k-1}, 1 \leq k \leq K$. Solving the system $R_{K} Q_{K}=P_{K}$, we express the flow rates through the outlet 
sections

$$
Q_{K}^{i}=\sum_{j=1}^{2^{K-1}} c_{i j}\left(P_{0}-P_{K}^{j}\right), \quad 1 \leq i \leq 2^{K-1}
$$

where $R_{K}^{-1}=\left(c_{i j}\right)_{1 \leq i, j \leq 2^{K-1}}$. Then, introducing the equivalent resistance

$$
R=\left(\sum_{i, j=1}^{2^{K-1}} c_{i j}\right)^{-1}
$$

it yields

$$
R Q_{0}=P_{0}-R \sum_{j=1}^{2^{K-1}}\left(\sum_{i=1}^{2^{K-1}} c_{i j}\right) P_{K}^{j}
$$

which means that the tree-like airway with $K$ generations behaves like a one-branch airway with equivalent resistance $R$ and equivalent outlet pressure

$$
R \sum_{j=1}^{2^{K-1}}\left(\sum_{i=1}^{2^{K-1}} c_{i j}\right) P_{K}^{j}
$$

Note that this equivalent pressure is a convex combination of the real outlet pressures $P_{K}^{j}$.

\subsection{Calculations by the electric analogy}

In practice, we avoid computing the reciprocal of $R_{K}$. We treat this bifurcation problem using an electric analogy: the pressure difference corresponds to the voltage drop, the flow rate to the electric current, and the flow resistance to the electric resistance. Observing that the daughter branches are branched together in parallel and then in series to the parent branch, we infer that in a two generation dichotomous airway tree the equivalent resistance is

$$
R_{k}^{i}+\left(\frac{1}{R_{k+1}^{2 i-1}}+\frac{1}{R_{k+1}^{2 i}}\right)^{-1}
$$

and the equivalent pressure is

$$
\left(\frac{1}{R_{k+1}^{2 i-1}}+\frac{1}{R_{k+1}^{2 i}}\right)^{-1}\left(\frac{P_{k+1}^{2 i-1}}{R_{k+1}^{2 i-1}}+\frac{P_{k+1}^{2 i}}{R_{k+1}^{2 i}}\right)
$$

To generalize these calculations to any type of dichotomous assymetric dissipative airway tree of $K$ generations, the algorithm consists in starting from the outlets and using the formula established for the bifurcation unit up to the inlet of the dissipative airway tree. The global resistance of a $K$ generations dissipative airway tree will be calculated first at the level of generation $K-1$ for $2^{K-2}$ bifurcation units. These global resistances calculated at the $K-1$ generation are used to calculate likewise the $2^{K-3}$ global resistances situated at generation $K-2$. The same process is applied recurcively in order to determine the global resistance of the whole dissipative airway tree. The values of the global resistances calculated at each generation level are stored in a $K 2^{K}$ matrix which is initially zero except in the $K$-th column, which is filled with the values of the generation $K$ resistances. The global pressure is calculated similarly once the resistance matrix is filled because the global pressure determination at a specific generation necessitates the knowledge of the corresponding global resistance. 

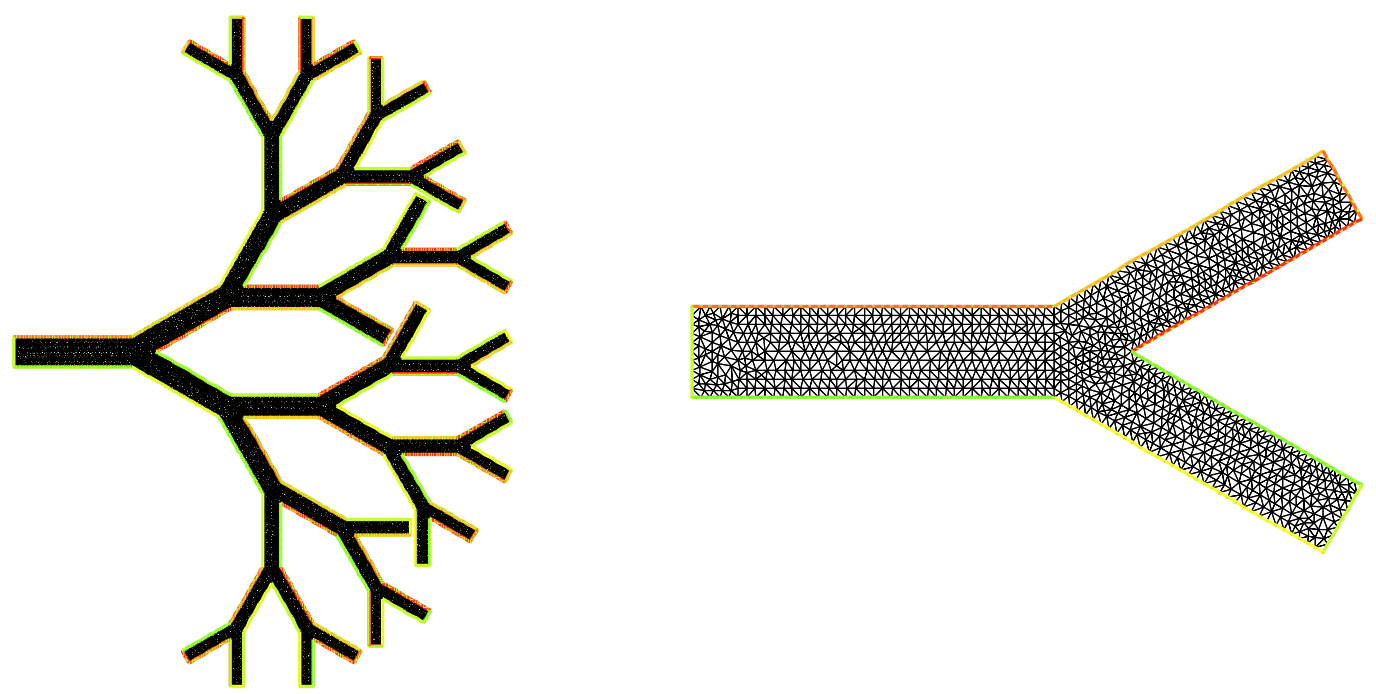

Figure 1. Whole and condensed trees

\section{Numerical Simulations}

\subsection{Two-dimensional simulations}

The Finite Element solver FreeFem++ (see [2]) has been used to run simulations both in a whole two dimensional 5-generation airway tree (with 26 oulets) and its corresponding condensed airway tree with a single bifurcation. The mesh of the condensed dissipative airway tree contains around 2200 tetrahedra while the mesh of the whole airway tree is composed of approximately 16000 tetrahedral elements. Our first step is to run simulations on the whole two dimensional (5-generation) airway tree. The fluid circulating is newtonian with unit viscosity and density. The boundary conditions applied on the inlet and on the outlets are of Neuman type. More precisely, these are homogeneous free on the inlet, non homogeneous on the outlets and we consider that the presure on each outlet is equal to -1 . A simulation has been performed with FreeFem ++ in the whole airway tree for a Stokes flow. The contours of the pressure intensity are presented on Figure 4.1.

Then, we perform simulations in the condensed airway tree. Since we cannot directly implement dissipative boundary conditions with FreeFem ++ , we use an optimization technique that allows us to calculate the pressure and velocity fields in the whole airway tree once the the pressure and velocity fields in the condensed airway tree are known. To achieve this goal, we proceed as follows: first, we consider an condensed airway tree, formed of a single bifurcation, in which the upper outlet is closed. Parabolic velocities are applied on the inlet and on the lower outlet. We solve numerically the Stokes problem associated with these boundary conditions.

The same simulations are then performed for the symmetric problem and this gives $u_{2}, p_{2}$. Next, we compute the numbers $\alpha_{1}$ and $\alpha_{2}$ satisfying

$$
u=\alpha_{1} u_{1}+\alpha_{2} u_{2} \text { and } p=\alpha_{1} p_{1}+\alpha_{2} p_{2}+\beta,
$$

together with $(u, p)$ is the solution of the Stokes problem for a condensed airway tree with particular boundary conditions. These are of dissipative essential type and are described in details in section (2.2). The global resistances Res 1 and Res 2 corresponding to both of the airway outlets of the condensed airway tree are respectively 126 and 139. Their calculations have been performed according to section (3). This also make it possible to calculate the global pressures corresponding to both of the airway outlets of the condensed airway tree. Therefore, we obtain a three equations system with three unknowns. The resolution of the previous system leads to 


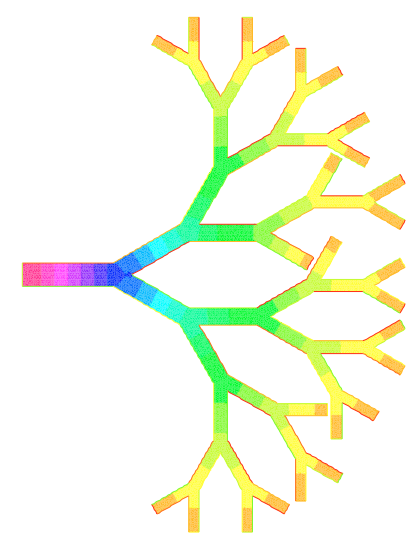

Figure 2. Pressure contours in the whole airway tree

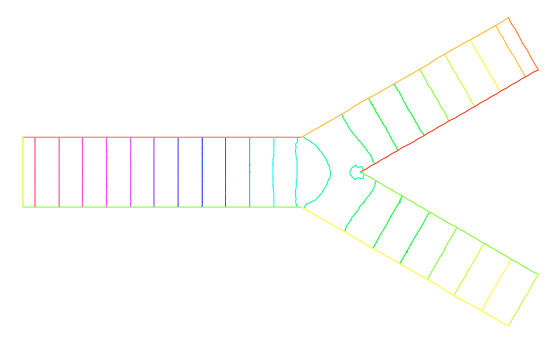

Figure 3. Pressure contours, Condensed airway tree

the values of $\alpha_{1}, \alpha_{2}$ and $\beta$. Finally, we numerically solve the Stokes problem for the whole airway tree with the boundary conditions obtained with $\alpha_{1}, \alpha_{2}$ and $\beta$. The contours of the pressure intensity are presented on Figure 3.

The following table gives the values of the flow values and pressure values for the condensed airway tree and for the whole airway tree at the first bifurcation level.

\begin{tabular}{|c|c|c|c|c|}
\hline & outlet flow rate (inf) & outlet flow rate (sup) & outlet pressure (inf) & outlet pressure (sup) \\
\hline condensed tree & 0.0033 & 0.0035 & -0.544 & -0.558 \\
\hline full tree & 0.0037 & 0.0039 & -0.576 & -0.588 \\
\hline
\end{tabular}

The results obtained in the condensed and the whole airway trees are relatively in good agreement since there is a $10 \%$ mistake on the values of the pressure and velocity. This error is mainly due to a relatively bad geometrical approximation of the bifurcation area, when we compute the equivalent resistance of a subtree. This aspect has to be improved in the future. 


\subsection{Three-dimensional simulations}

Finite element solvers FIDAP and a home-made code (which we shall refer to as FEM) have been used to run simulations both in a whole three dimensional multiple (two or three) generations airway tree and its corresponding condensed airway tree formed of a single bifurcation.

Dissipative boundary conditions have been directly implemented in FEM while this could not be done with the commercial code FIDAP. For this latter code, the outlet airway branches of the condensed airway tree have been extended by a cylindrical tube, which is assumed to be filled with a fictitious fluid of viscosity $\tilde{\mu}$. Using again Poiseuille's law the flow resistance in this extension of length $L$ and diameter $D$ is $R=128 \tilde{\mu} L /\left(\pi D^{4}\right)$. The value of $\tilde{\mu}$ is chosen in such a way that $R$ matches the equivalent resistance of the condensed airway tree. The mesh of the condensed dissipative airway tree contains about 1000 tetrahedra while the mesh of the whole airway tree is composed of approximately 4000 tetrahedra.

The fluid circulating both in the whole and the condensed airway tree is newtonian with a unit viscosity and density. Velocities or pressure are applied on the inlet of the whole airway tree and free boundary conditions are imposed on the outlets. The boundary conditions applied to the condensed airway tree are the same on the inlet and dissipative boundary conditions equivalent to the influence of the dissipative zone of the whole airway tree were imposed on the outlets. Boundary conditions will be described specifically for each of the following test cases. The simulations are performed for steady conditions. In this part, all the units are S.I.

\subsubsection{Stokes flow}

Tests have been performed in the case of homogeneous and non-homogeneous outlet conditions. The following simulation has been performed with a uniform pressure (the value is set at 10) at the inlet and homogeneous free outlets. The boundary conditions applied to the condensed airway tree are the same on the inlet and dissipative boundaries are imposed on the outlets. The global resistances Res1 and Res2 corresponding to both of the airway outlets of the condensed airway tree are respectively 15.8 and 14.2. They have been calculated according to section 3 . They have different values due to the asymmetry of the complete airway tree.

The pressure field calculated numerically with FEM in the condensed airway tree (cf. figure 4.2.1) and the one which corresponds to the whole airway tree (cf. figure 4.2.1) are qualitatively in good agreement. The pressure difference between the inlet and each of the outlets is around 7 in the condensed airway tree and the corresponding value is qualitatively the same in the whole airway tree.

A simulation has been performed with Fidap in the whole airway tree. A parabolic velocity with a unit intensity profile is imposed on the inlet and homogeneous free outlets are applied on all the outlets except one which is non homogenous and equals 30. Hence, when the flow goes through this non homogeneous free outlet, it is actually directed towards the inlet of the airway tree. The same inlet boundary condition is applied on the inlet of the condensed airway tree and on the inlet of the whole airway tree. The virtual viscosities $\mu 1$ and $\mu 2$ corresponding to the global resistances Res1 and Res2 imposed for the previous FEM calculation equal respectively 5.59 and 6.22 .

The pressure values obtained on reference sections located at the same level in the whole and the condensed airway tree can be compared quantitatively. The two chosen reference sections called section 1 and section 2 are located at the interface between the unit viscosity zone and the virtual viscusity $\mu 1$ or $\mu 2$ zones. The error between the pressure calculated over corresponding sections in the whole and the condensed airway trees is lower than $2 \%$ as is illustrated on the following table.

\begin{tabular}{|c|c|c|}
\hline & section 1 & section 2 \\
\hline condensed airway tree & 24.5 & 17.1 \\
\hline whole airway tree & 24.4 & 16.8 \\
\hline
\end{tabular}

The qualitative and quantitative results obtained in the condensed and the whole airway trees are in good agreement and the calculation duration is three times lower in the condensed airway tree than in the whole airway tree. Logically, the more generations are taken into account in the dissipative boundary condition, the more the calculation duration should be decreased in the condensed airway tree with respect to the whole airway 


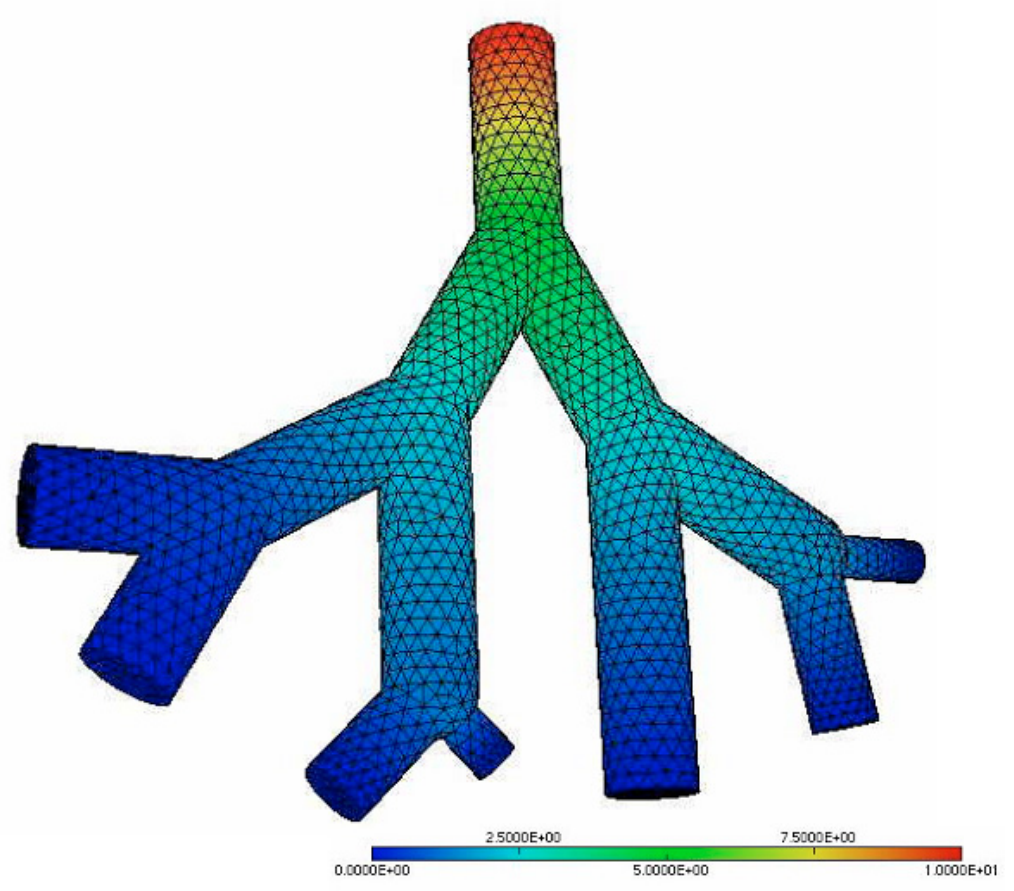

Figure 4. Pressure contours, Whole airway tree

tree. This is encouraging for future studies involving more realistic airway trees. Detailed comparisons between FIDAP and MISTRAL results are currently being performed.

\subsubsection{Navier-Stokes flow}

The computational model is the same as in the previous section (with homogeneous free outlets) except that the convective term of the Navier-Stokes flow is added to the previous Stokes flow model. The influence of mesh refinements on the calculations in the condensed airway tree is studied and results obtained with the initial mesh with 1000 tetrahedra and with a four time more refined mesh are compared. Likewise, two preliminary tests have been performed first to study the effect of the length of the extensions where the virtual viscosity is imposed and second, to determine the influence of the Reynolds number calculated at the location where the dissipative boundary conditions are imposed. In this latter test case, the pressure difference imposed between the inlet and the outlets of the whole airway tree was between 30 and 750, which corresponds to a Reynolds number evoluating from 1 to 45 over the inlet. The presurre and velocity fields calculated in the whole and the condensed airway tree are well superimposed and results can be improved using a finer mesh of longer extensions.For instance, the value of the mean pressure on a test section located half a diameter upstream from the interface between the unit viscusity and $\mu 2$ are indicated for each type of tested meshes in the following table.

\begin{tabular}{|l|c|c|}
\hline Pressure difference between the inlet and the outlets & 30 & 750 \\
\hline whole airway tree & 10.4 & 280 \\
\hline condensed airway tree - number of tetrahedra $=1000-\mathrm{L}=1$ & 10.6 & 292 \\
\hline condensed airway tree - number of tetrahedra $=4000-\mathrm{L}=1$ & - & 288 \\
\hline condensed airway tree - number of tetrahedra $=4000-\mathrm{L}=2$ & - & 283 \\
\hline
\end{tabular}

The more the pressure difference between the inlet and the outlets of the whole airway tree increases, the more the pressure value over the test section are not homogeneous since non linear effects of the Navier-Stokes 


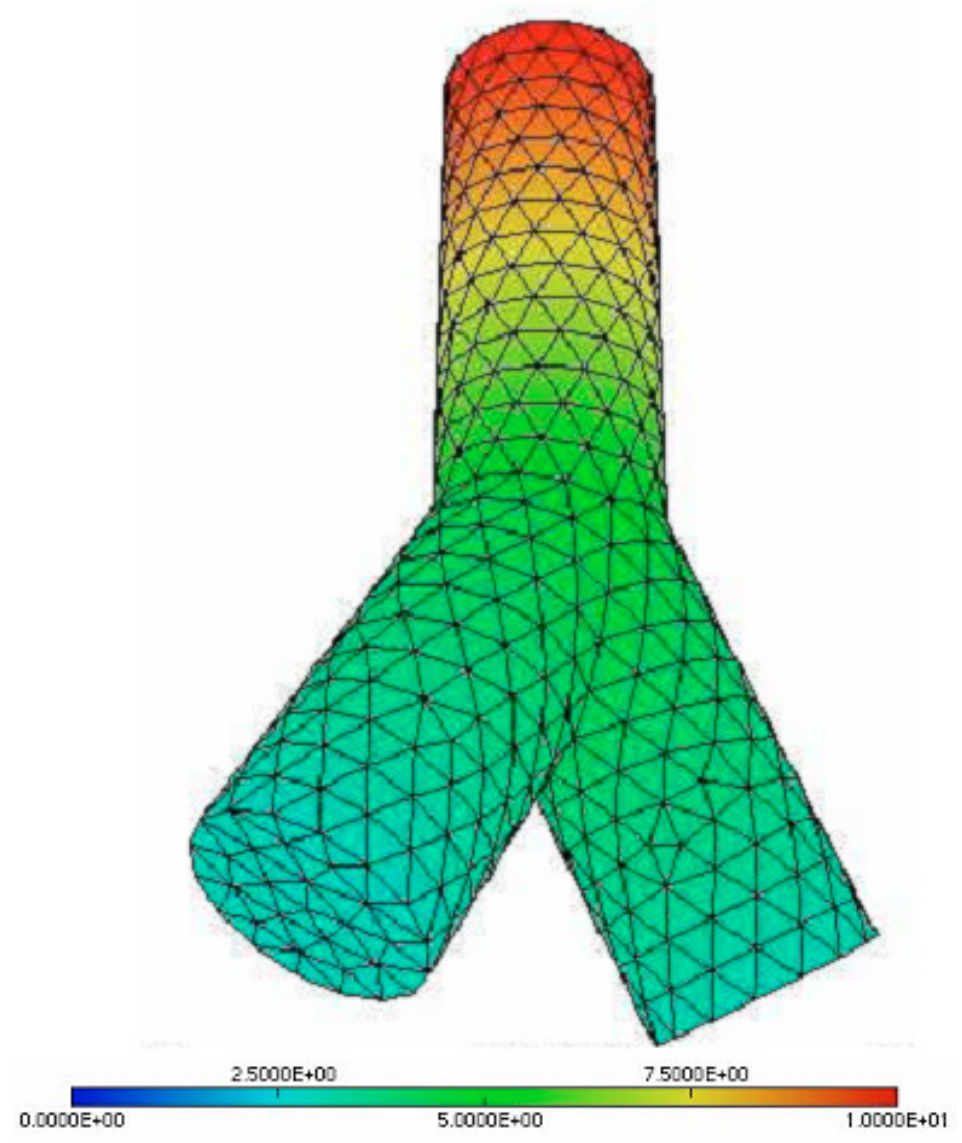

Figure 5. Pressure contours, Condensed airway tree

equation become more important and the more the mesh quality and tube length plays an important role. These results correspond to those observed on other sections. This study is currently being pursued especially in the case when the pressure difference between the inlet and the outlets is more important than in the test cases performed here. The ultimate goal is the determination of the Reynolds number limit under which the dissipative boundary conditions exposed in this paper can be trusted while making a reasonable mistake

\subsubsection{Analytical reconstruction of the flow rates}

Since the geometry of the whole airway tree is supposed to be known, it is possible to infer each branch flow rate in the whole airway tree based on the pressure values numerically calculated on the outlets of the condensed airway tree. The flow rates numerically calculated on the outlets of the whole airway tree and those reconstructed analytically from the pressure values obtained of the outlets of the condensed airway tree are both presented in Figure 6. Quantitatively, the flow rates on the outlet bronchioles of the whole airway tree calculated numerically and analytically are the same with a $5 \%$ estimated error.

\section{Conclusion}

The multiplicity of the simulation test cases performed with three different finite element codes, the promising comparison between the whole and its corresponding condensed airway tree results are very encouraging for 

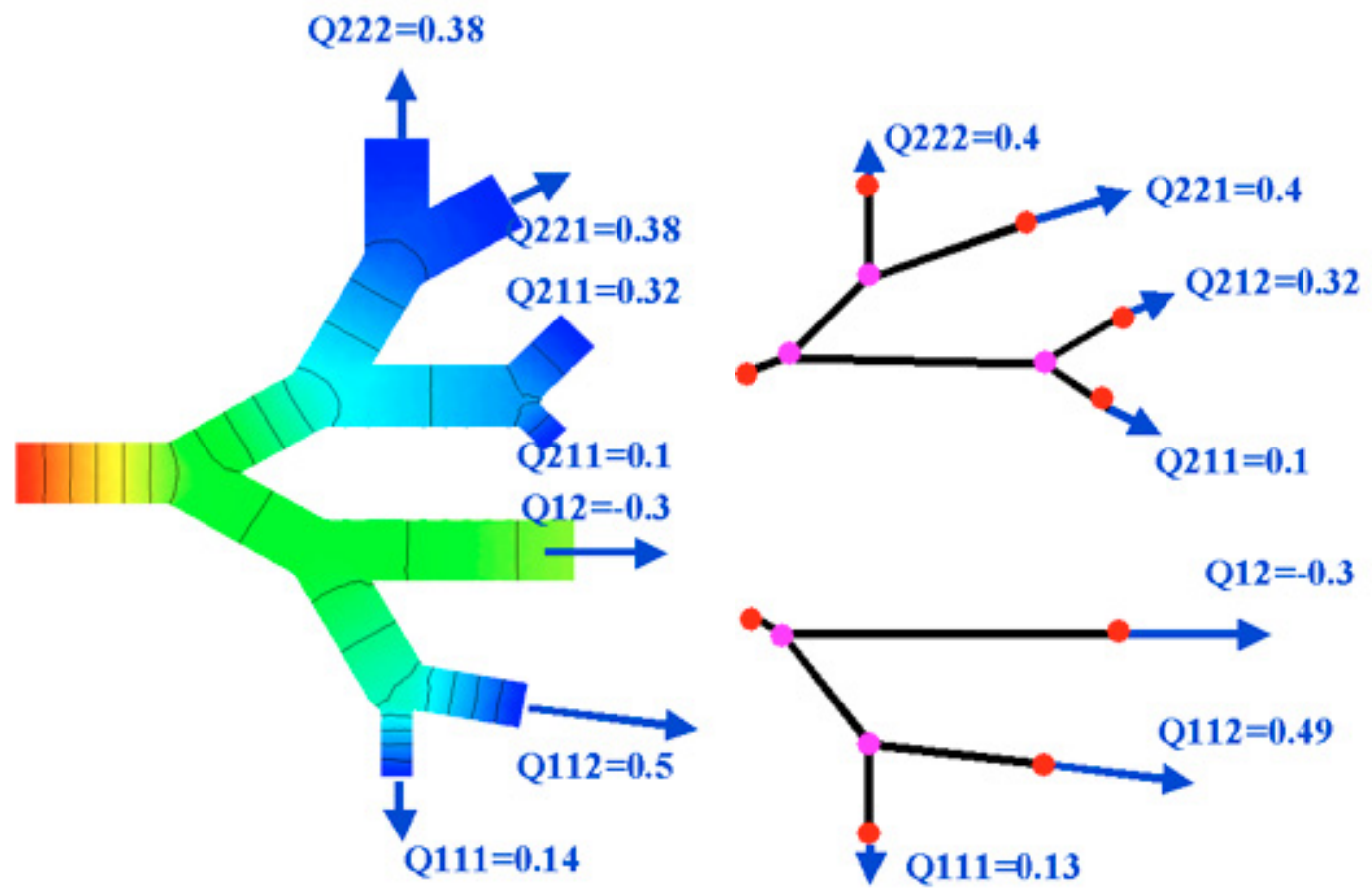

FiguRE 6. Flowrates (left: full simulation; right: for the condensed tree)

future studies on dissipative boundary conditions. Air flow simulations in proximal human airways reconstructed from the trachea to the 5th generation based on medical imaging such as computerized tomography would combine Navier-Stokes simulations in proximal airways to dissipative boundaries that would take into account the complex downstream pulmonary tree. Calculation duration in this proximal airway model with dissipative boundaries would be significantly lower than in the whole realistic airway tree from the trachea down to the 23rd generation and similar to calculation duration in the proximal airways with homogeneous free outlets. Finally, dissipative boundary conditions could be used to run complex time-dependent simulations. Hence, simulating the alveoli opening and closing during the respiratory cycle using transitory dissipative boundary outlets would be possible.

\section{REFERENCES}

[1] C. Conca, F. Murat, O. Pironneau, Stokes and Navier-Stokes equations with boundary conditions involving the pressure, Japan. J. Math., Vol. 20, No. 2, 1994.

[2] http://www.freefem.org

[3] L. Formaggia, J.-F. Gerbeau, F. Nobile, A. Quarteroni, Numerical treatment of defective boundary conditions for the NavierStokes equations, SIAM J. Number. Anal. , Vol. 40, No. 1, 376-401.

[4] C. Grandmont, Y. Maday, B. Maury, A multiscale/multimodel approach of the respiration tree, à paraître.

[5] V. Girault, P.-A. Raviart, Finite Element Approximation of the Navier-Stokes Equations, Lecture Notes in Mathematics, Vol 749 Springer Verlag, Berlin, 1979.

[6] B. Mauroy, M. Filoche, J.S. Andrade Jr., B. Sapoval, Interplay between flow distribution and geometry in an airway tree, Phys. Rev. Lett. 90, 148101-1-148101-4- (2003).

[7] Patrick Ciarlet Jr, Système de Stokes avec flux de vitesse et pression imposés, C. R. Acad. Sci. Paris, Ser. 1337 (2003) $119-124$.

[8] A. Veneziani, C.Vergara, Flow rate defective boundary conditions in haemodynamics simulations, Int. J. Numer. Meth. Fluids, 2000, 00, 1-6. 